\title{
A Mechanism of Rapidly Reversible Cerebral Ventricular Enlargement Independent of Tissue Atrophy
}

\author{
Natalie M Zahr, ${ }^{*, 2}$, Dirk Mayer ${ }^{2,3}$, Torsten Rohlfing ${ }^{2}$, Juan Orduna ${ }^{2}$, Richard Luong ${ }^{4}$, Edith V Sullivan' and \\ Adolf Pfefferbaum ${ }^{1,2}$ \\ 'Department of Psychiatry and Behavioral Sciences, Stanford University School of Medicine, Stanford, CA, USA; ${ }^{2}$ Neuroscience Program, \\ SRI International, Menlo Park, CA, USA; ${ }^{3}$ Lucas MRS/I Center, Department of Radiology, Stanford University, Stanford, CA, USA; ${ }^{4}$ Department of \\ Comparative Medicine, Stanford University, Stanford, CA, USA
}

\begin{abstract}
Ventricular enlargement, a common in vivo marker of aging, disease, and insult, is presumed to reflect atrophy of surrounding brain regions. Pathological mechanisms underlying ventricular enlargement, however, are likely specific to the condition under investigation. Here, multimodal imaging, incorporating structural magnetic resonance imaging (MRI), MR spectroscopy (MRS), and diffusion weighted imaging (DWI), was used in rats exposed to binge ethanol (EtOH) to provide insight into a mechanism of reversible ventricular enlargement. During intoxication, MRI revealed expansion of ventricles, but volume changes in dorsal or ventral hippocampi, caudateputamen, or thalamus were not detectible. MRS of whole-brain parenchyma showed decreases in N-acetylasparate (NAA) and tissue water T2, and increases in choline-containing compounds (Cho). DWI showed decreased diffusivity selective to the thalamus. All MR parameters returned to baseline with 7 days of recovery. Rapid recovery of ventricular volume and the absence of detectable tissue volume reductions in brain regions adjacent to ventricles argue against atrophy as a mechanism of ventricular expansion. Decreased tissue water T2 and decreased thalamic diffusivity suggest lower tissue water content and a role for both NAA and Cho, as osmolytes is proposed. Together, these data support a model of fluid redistribution during acute $\mathrm{EtOH}$ intoxication and recovery to account for rapid ventricular volume changes.

Neuropsychopharmacology (2013) 38, II21-II29; doi: I0.1038/npp.2013.1 I; published online 27 February 2013
\end{abstract}

Keywords: binge; alcoholism; ventriculomegaly; magnetic resonance (MR) imaging; MR spectroscopy; diffusion weighted imaging

\section{INTRODUCTION}

Cerebral ventricular enlargement occurs in numerous conditions including normal aging (Pfefferbaum et al, 2006), traumatic brain injury (Bigler and Maxwell, 2011), Alzheimer's disease (Fox and Schott, 2004), and schizophrenia (Sayo et al, 2012). The frequency and consistency of this finding may reflect the ease and reliability of lateral ventricle measurement with ultrasound, computed tomography, and magnetic resonance imaging (MRI). Although a sensitive and prevalent in vivo marker of CNS pathology, ventriculomegaly is reversible in certain conditions (eg, following recovery from alcoholism, anorexia nervosa (Enzmann and Lane, 1977), and prolonged steroid use (Bentson et al, 1978)), thereby challenging the hypothesis that ventricular enlargement predicts neuronal loss (Symonds et al, 1999).

\footnotetext{
*Correspondence: Dr NM Zahr, Psychiatry \& Behavioral Sciences, Stanford University School of Medicine, 401 Quarry Road, Stanford, CA 94305, USA, Tel: + I 650859 5243, Fax: + I 650859 2743,

E-mail: nzahr@stanford.edu

Received 5 November 2012; revised 2 January 2013; accepted 4 January 2013; accepted article preview online 10 January 2013
}

In a rodent model of alcoholism, binge ethanol (EtOH) exposure to blood alcohol levels (BALs) of $\sim 250 \mathrm{mg} / \mathrm{dl}$ revealed ventricular expansion to $\sim 115 \%$ of baseline that was completely reversed with 7 days of recovery (Zahr et al, 2010). Rapid expansion and recovery of ventricular volume suggests lack of permanent tissue damage. Instead, the etiology of this ventricular enlargement likely reflects biological and physiological processes unique to high $\mathrm{EtOH}$ treatment but that can be deciphered using multimodal MRI.

The power of MRI rests in its ability to garner in vivo quantitative measures that permit longitudinal tracking of disease progression, intervention, and recovery. Conventional MRI reveals anatomical features of the brain and is typically used to quantify brain tissue volume and shape. Beyond structural MRI, additional MR modalities provide unique metrics that enable the study of distinct biochemical and physiological phenomena. Biochemical evaluation of tissue is accomplished with MR spectroscopy (MRS). Levels of the predominant MRS signal from $\mathrm{N}$-acetylaspartate (NAA) are irreversibly reduced in diseases in which neuronal loss incontrovertibly occurs (eg, epilepsy or ischemia) (Bengzon et al, 2002; Jackson et al, 2011), but are only transiently reduced in conditions where recovery is possible (eg, following detoxification from 
chronic alcoholism) (Bendszus et al, 2001). The MRS signal composed of choline-containing compounds (Cho) is elevated in multiple sclerosis (Butzkueven et al, 2008) and often interpreted as representing neuroinflammation (De Stefano et al, 2005) or demyelination (Mader et al, 2008). Diffusion weighted imaging (DWI) assesses water diffusion in brain tissue, revealing alignment of cellular structures and quality of tissue microstructure, including the condition of myelin.

Using multimodal imaging (MRI, MRS, and DWI), we sought to determine mechanisms underlying acute reversible ventricular enlargement caused by binge EtOH exposure. Accordingly, rats underwent MR examination before, during, and after recovery from binge $\mathrm{EtOH}$ exposure (Majchrowicz, 1975). We expected ventriculomegaly, as revealed by MRI to be accompanied by decreases in NAA and increases in Cho in brain parenchyma measured with MRS. We also predicted that diffusivity in tissue adjacent to ventricular margins would be transiently altered. We tested the hypothesis that reversible ventricular expansion observed with MRI in response to binge EtOH treatment is compensatory for changes in tissue water content, quantifiable with MRS and DWI.

\section{METHODS}

\section{Animals}

The study group initially included 22 male, wild-type Wistar rats (Charles River Laboratories), singly housed with free access to food and water with lights on for $12 \mathrm{~h}$ starting at 08:00 hours. At baseline (ie, the first scan), rats weighed $334.83 \pm 3.7 \mathrm{~g}$. The Institutional Animal Care and Use Committees at SRI International and Stanford University approved all procedures.

\section{Treatment}

After baseline scanning, 11 rats were assigned to the EtOH group and received an initial 'loading' dose of $5 \mathrm{~g} / \mathrm{kg} 20 \%$ $\mathrm{EtOH} \mathrm{w} / \mathrm{v}$ via oral gavage, then a maximum of $3 \mathrm{~g} / \mathrm{kg}$ every $8 \mathrm{~h}$ for 4 days. On each of the 4 days, animals were weighed and tail vein blood samples were collected within $4 \mathrm{~h}$ of the first dose (ie, 11:00 hours) to determine BALs in plasma assayed for alcohol content based on direct reaction with the enzyme alcohol oxidase (Analox Instruments, UK).

EtOH was administered according to body weight, BALs, and behavioral evidence for intoxication. Daily doses averaged $7.21 \pm .32 \mathrm{~g} / \mathrm{kg} /$ animal (cumulative dose across 4 days $=28.85 \pm 1.26 \mathrm{~g} / \mathrm{kg} / \mathrm{animal}$ ) at a daily volume averaging $8.87 \pm .46 \mathrm{ml} /$ animal. Control (Dex) animals received volumes of $5 \%$ dextrose equivalent to $3 \mathrm{~g} / \mathrm{kg} \mathrm{EtOH}$ (daily volume averaging $11.86 \pm .43 \mathrm{ml} / \mathrm{animal}$ ) at comparable times to the experimental animals, ie, at about 07:00, 15:00, and 23:00 hours. Because the EtOH dose was titrated based on BALs and behavioral intoxication states, while dextrose was given at a constant volume based on $3 \mathrm{~g} / \mathrm{kg}$ $\mathrm{EtOH}$ per day, the Dex group received a greater intragastric fluid load than the EtOH group $(t(19)=4.75, P=0.0001)$.

\section{MR Scanning Procedures and Data Analysis}

Schedule. Animals were scanned at baseline, after 4 days of treatment (binge scan; within $7.5 \mathrm{~h}$ of the last dose), and after 7 days of recovery. One EtOH rat received a baseline scan but died during EtOH treatment; two $\mathrm{EtOH}$ rats received baseline and binge scans, but died before the recovery scans. One Dex rat did not receive a baseline scan; another Dex rat had values for MR variables that were more than eight SD values from the mean, suggesting scanner failure. The remaining eight $\mathrm{EtOH}$ and nine Dex rats had three complete scans, and ten EtOH and ten Dex rats had at least two scans.

Anesthesia and Monitoring. Animals were held in a MR-invisible structure providing support for a radiofrequency (RF) coil and a nose cone for delivery of isoflurane anesthesia (1.5-3.5\%) and oxygen $(1.5 \mathrm{l} / \mathrm{min})$ (Adalsteinsson et al, 2004). For each rat, blood oxygen saturation, pulse rate, rectal temperature, and respiration were monitored throughout the $\sim 2 \mathrm{~h}$ MR experiment.

MRI Acquisition. The experiments were conducted on a clinical 3T GE Signa MR scanner equipped with a highstrength insert gradient coil (peak strength $=600 \mathrm{mT} / \mathrm{m}$, peak slew rate $=3200 \mathrm{~T} / \mathrm{m}$ per s, Chronik et al, 2000; Pfefferbaum et al, 2004). The gradient system operates at maximum amplitude of $500 \mathrm{mT} / \mathrm{m}$ with a slew rate of $1800 \mathrm{mT} / \mathrm{m}$ per ms. A custom-made rat brain quadrature head coil $(\varnothing=44 \mathrm{~mm})$ was used for both RF excitation and signal reception. A gradient-recalled echo localizer scan was used to position the animals in the scanner and for graphical prescription of the subsequent scans. Highresolution, dual-echo, fast spin-echo (FSE) images were acquired in the rat axial plane, coronal to the magnet system bore $(\mathrm{TE} 1 / \mathrm{TE} 2 / \mathrm{TR}=12 / 60 / 5000 \mathrm{~ms}$, field of view $(\mathrm{FOV})=64 \times 48 \mathrm{~mm}^{2}, \quad 256 \times 192$ matrix, echo train length $=8,26$ slices, $0.7 \mathrm{~mm}$ thick, $0 \mathrm{~mm}$ separation, inplane resolution $=0.25 \times 25 \mathrm{~mm}^{2}$, two separate acquisitions each with two NEX). For details regarding MR Image Postprocessing, please see supplementary material.

MRS Acquisition. FSE images were used to prescribe a voxel $\left(9 \times 7 \times 9 \mathrm{~mm}^{3}=567 \mathrm{~mm}^{3}\right)$ fitting within but including as much of the brain as possible while excluding the olfactory bulbs and cerebellum. Single-voxel spectroscopic data were acquired with point-resolved spectroscopy $(\mathrm{TE} / \mathrm{TR}=133 / 2283 \mathrm{~ms}, \mathrm{NEX}=128)$ (Bottomley, 1984) preceded by a three-pulse chemical shift selective sequence for water suppression. Additionally, data without water suppression from the same voxel were acquired at multiple TEs (TE ranging from 36.6 to $241.4 \mathrm{~ms}, 12.8 \mathrm{~ms}$ increment, $\mathrm{TR}=2 \mathrm{~s}$ ) to measure tissue water content for normalization of metabolite signal intensities to the amount of tissue water in the voxel (for details, see Zahr et al, 2009). The three singlet resonances (NAA, total creatine ( $\mathrm{tCr}$ ), and Cho) were fit simultaneously, and the $\mathrm{EtOH}$ and Glx (combined resonances of glutamate and glutamine) resonance fit independently, with a Gaussian function within a $\pm 7.95 \mathrm{~Hz}$ window using a downhill simplex method (IDL AMOEBA). The integrated area under the fitted Gaussian was used for quantification. The quality of the spectra allowed evaluation of signals of the major proton metabolites: NAA (2.01 p.p.m.), tCr (3.03 p.p.m.), Cho (3.20 p.p.m.), Glx (3.78 p.p.m.), and EtOH (1.18 p.p.m.) (Figure 1). 

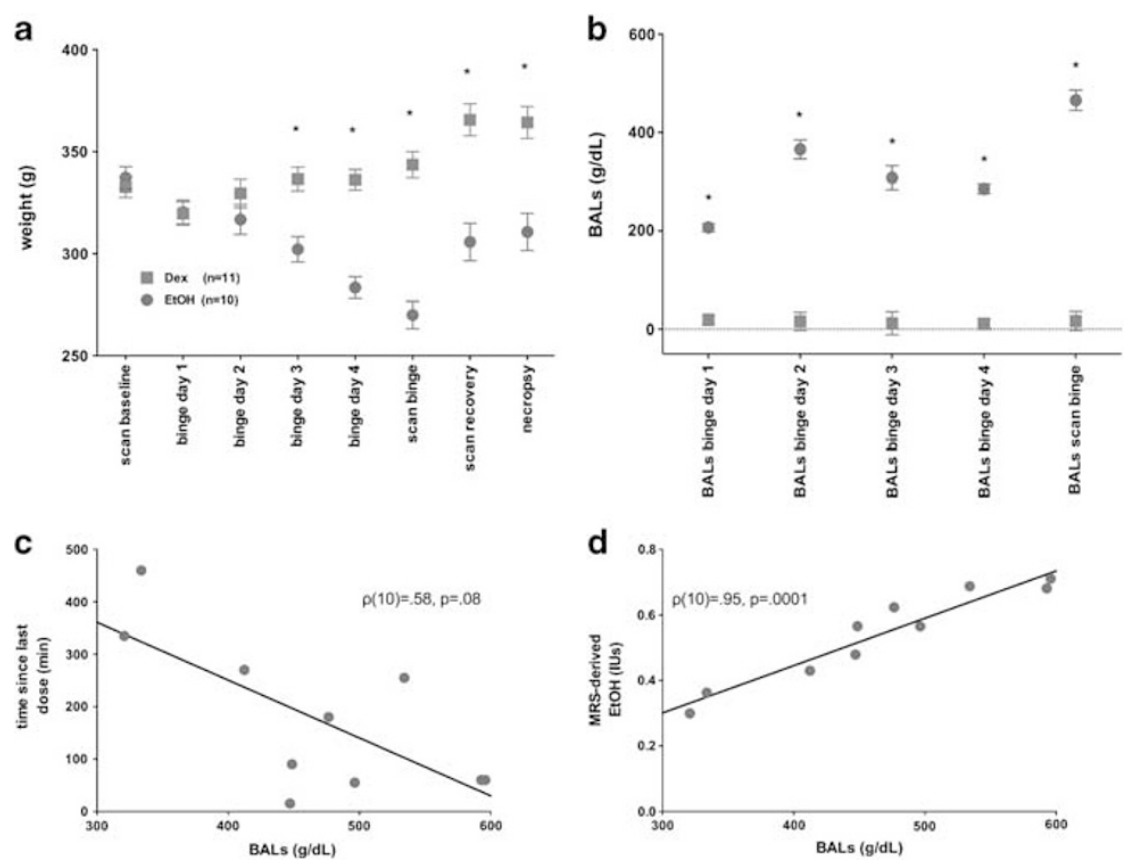

Figure I (a) Weights of EtOH (red circles) and Dex (gray squares) animals across the course of the experiment. (b) BALs on each day of treatment and immediately following the binge MR scan. (c) Correlation between BALs and time of last EtOH dose. (d) Correlation between BALs and in vivo brain EtOH measures by MRS. For weights * $P \leqslant 0.005$, for *BALs $P \leqslant 0.000$ I. BALs, blood alcohol levels; Dex, dextrose; EtOH, ethanol; MR, magnetic resonance; MRS, MR spectroscopy. Note: 'binge scan' weight and BAL measurements took place within $9.5 \mathrm{~h}$ of the last EtOH dose (ie, at end of scan on day 5), while weight and BAL measurements during treatment were collected at 11:00 (ie, 1 I:00 of day 4). The color reproduction of this figure is available on the Neuropsychopharmacology online.

DWI Acquisition. The DWI pulse sequence used a $\mathrm{FOV}=32 \times 32 \mathrm{~mm}^{2}$ and $\mathrm{TE} / \mathrm{TR}=32 / 2000 \mathrm{~ms}$. Multi-slice data were acquired in rat coronal (scanner axial) orientation at an in-plane resolution of $0.5 \mathrm{~mm}$ with and without diffusion weighting. The slice prescription was the same as for FSE imaging. Diffusion weighting was performed in six noncollinear directions $(+x+y,+y+z,+x+z,-x+y$, $-x+z,+x-z)$ with a b-value of $1700 \mathrm{~s} / \mathrm{mm}^{2}$; the same six directions with opposite polarity were acquired to compensate for the cross-terms caused by both imaging and crusher gradients (Neeman et al, 1991). Frequency-selective lipid suppression and outer-volume suppression modules preceded the imaging sequence. Saturation bands were placed at the superior and inferior edge of the FOV; six averages per acquisition were collected with the positive and negative polarity gradient scheme and repeated 12 times for a total DWI acquisition time of $45 \mathrm{~min}$.

DWI Analysis. DWI quantification was preceded by peranimal motion correction based on rigid registration of the first $b=0$ image to each of the subsequent $b=0$ images. From the motion-corrected images, diffusion tensors were then reconstructed using the Camino software toolkit (Cook et al, 2006). From the diffusion tensors, the scalar diffusion measures fractional anisotropy, mean diffusivity (MD), longitudinal diffusivity $(\lambda 1)$, and transverse diffusivity $(\lambda \mathrm{T})$ were then extracted, also using Camino. MD was the primary metric for assessment of tissue water content.

\section{Behavioral and Liver Analysis}

Please see Supplementary material.

\section{Statistical Analysis}

Group differences were tested with two-group (Dex vs EtOH)-by-three time-point (baseline, post-gavage, and recovery) repeated-measures analyses of variance (ANOVA); where appropriate, Greenhouse-Geiser (GG) correction was applied. Only group effects and group-by-session interactions were of interest to this analysis. Follow-up betweengroup and within-group differences were determined by two-tailed $t$-tests. Because of the small sample size, within-group comparisons were also evaluated using Spearman's $\rho$ (rank order correlation). Simple regressions evaluated correlations.

\section{RESULTS}

\section{Binge EtOH Affects Weight}

Figure 1a presents weights per group at each scan, during each day of treatment, and at study termination. Group differences in body weight were evident from day 3 of treatment $(t(21)=4.02, P=0.0007)$ and persisted through the remainder of the study. At the binge scan, the EtOH group weighed $21.5 \%$ less than the Dex group. Although the EtOH group gained weight between the binge and recovery scans $(t(7)=3.33, \quad P=0.01 ; Z=15, \quad P \leqslant 0.04)$, a weight difference endured, such that the EtOH group weighed $16.4 \%$ less than the Dex group at the recovery scan and $15 \%$ less at study termination.

Figure $1 \mathrm{~b}$ presents BALs on each day of treatment and at the binge scan. Across the 4 days of treatment, the $\mathrm{EtOH}$ animals averaged BALs of $291.72 \pm 7.6 \mathrm{mg} / \mathrm{dl}$; BALs from blood samples collected immediately after the binge 
scan, within $7.5 \mathrm{~h}$ of the last dose of EtOH averaged $465.84 \pm 20.63 \mathrm{mg} / \mathrm{dl}$ and showed a trend towards correlating with time as the last $\mathrm{EtOH}$ dose given (Figure 1c, $\rho(10)=0.58, P=0.08)$. MRS-derived EtOH levels were highly correlated with BALs collected immediately after the binge scan (Figure $1 \mathrm{~d}, \rho(10)=.95, P=0.0001$ ).

For details regarding alcohol effects on behavior and liver, please see Supplementary material.

\section{Binge EtOH Reversibly Increases Ventricular Volume}

A two-group, repeated-measures ANOVA yielded a group-by-time interaction $(\mathrm{F}(2,30)=33.42, \quad P=0.0001$, $\mathrm{GG}=0.0001)$, indicating that EtOH exposure modified ventricular volume (Figure 2). At the binge scan, ventricular volume in the EtOH group had increased from baseline $(t(9)=7.26, P=0.0001)$ and was greater than in the Dex group $(t(18)=5.53, P=0.0001)$. At recovery, ventricular volume no longer differentiated the groups: the EtOH group evidenced a significant ventricular volume decrease between the binge and recovery scans $(t(7)=6.00, P=0.0005)$, achieving full recovery.

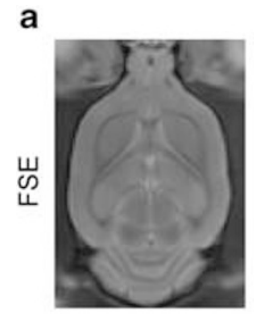

Lateral Ventricles
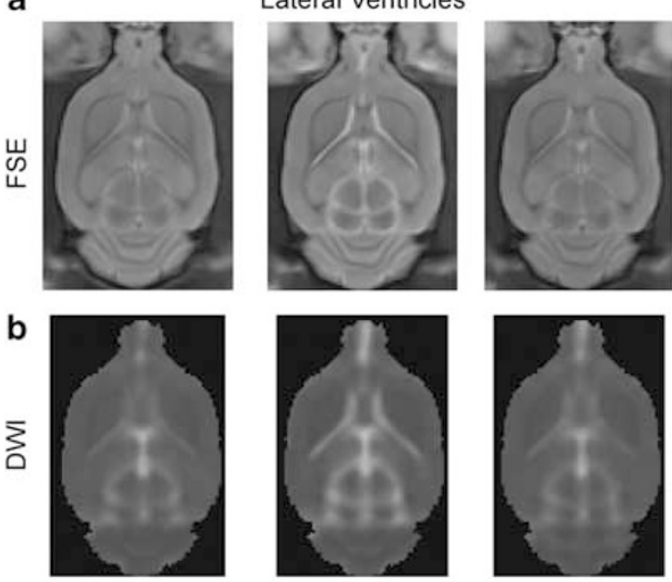

baseline

binge

recovery

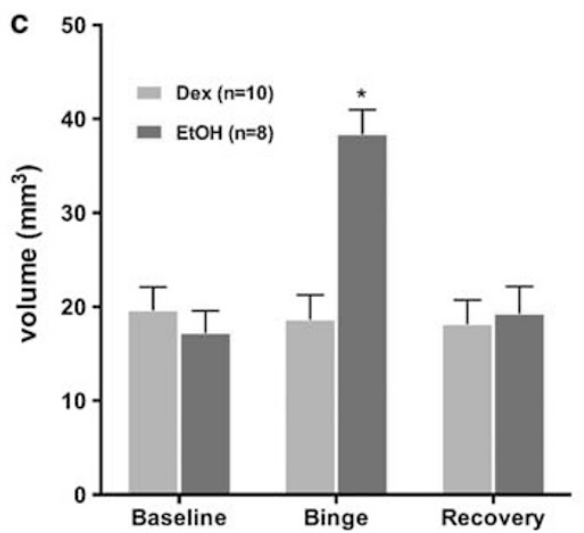

Figure 2 (a) FSE and (b) DWI images from an exemplary EtOH animal at each of the three scans demonstrating ventricular enlargement at the binge scan. (c) Quantification of ventricular volume in $\mathrm{EtOH}$ (red) and Dex (gray) animals based on DWI images at each of the three times points. Dex, dextrose; DWI, diffusion weighted images; EtOH, ethanol; FSE, fast spin-echo images; $* P \leqslant 0.05$. The color reproduction of this figure is available on the Neuropsychopharmacology online.

\section{Binge EtOH Doesnot Produce Detectable Volume Changes Of Selective Brain Regions}

Volumes of the dorsal and ventral hippocampi, caudateputamen, and thalamus were measured for potential increases or decreases in response to binge EtOH treatment. Two-group, repeated-measures ANOVAs failed to reveal significant effects of binge EtOH treatment on volumes of the dorsal hippocampus $(\mathrm{F}(2,30)=0.2774, \quad P=0.7597)$, ventral hippocampus $(\mathrm{F}(2,30)=0.6251, P=0.5420)$, caudate-putamen $(\mathrm{F}(2,30)=3.2866, P=0.0512)$, or thalamus $(\mathrm{F}(2,30)=2.132, P=0.1362)$ (Supplementary Figure S2).

\section{Binge EtOH Reversibly Modifies Select Proton Metabolite Levels}

A two-group, repeated-measures (three scan sessions, four metabolite, ie, NAA, tCr, Cho, and Glx) ANOVA revealed a group-by-metabolite-by-time interaction $(\mathrm{F}(6,96)=11.01$, $P=0.0001, G G=0.0001)$ indicating a significant $\mathrm{EtOH}$ effect on metabolite levels. Follow-up two-group-by-3 scan session ANOVAs for each metabolite separately demonstrated significant group-by-time interactions for NAA $(\mathrm{F}(2,32)=12.50, P=0.0001, \mathrm{GG}=0.0001)$ and Cho $(\mathrm{F}(2,32)=4.42, P=0.0202, \mathrm{GG}=0.0263)$ (Figure 3). Four days of binge EtOH treatment resulted in lower NAA $(t(19)=6.23, P=0.0001)$ and higher Cho $(t(19)=2.80$, $P=0.0114$ ) in the EtOH than the Dex group (Figure 3). Within the EtOH group, changes between baseline and binge scans were significant for NAA (decreased; $t(9)=4.60, \quad P=0.0013 ; \quad \rho(10)=.68, \quad P=0.03)$ and Cho (increased; $t(9)=2.65, P=0.01 ; \rho(10)=0.07, P=0.85)$. A family-wise Bonferroni correction for four metabolites and three time points with $\alpha=.05$ would require a directional hypothesis as significant with $P \leqslant 0.0008$, and a nondirectional test of significance at $P \leqslant 0.004$; thus, even with a twotailed Bonferroni correction for multiple comparisons, the decrease in NAA was significant between the groups.

After 7 days of recovery, metabolite levels no longer distinguished the groups. Within the $\mathrm{EtOH}$ group, the changes between the binge and recovery scan indicated a significant increase in NAA $(t(7)=2.69, P=0.0309 ; \rho(8)=$ $0.38, P=0.35)$ and decrease in Cho $(t(7)=2.81, P=0.0263$; $\rho(8)=0.45, P=0.2604)$ such that levels of these metabolites at recovery were not different from Dex animals.

Quantification of MRS-derived EtOH $(\mathrm{F}(2,32)=140.94$, $P=0.0001)$ showed high levels in the EtOH group at the binge relative to baseline $(t(9)=11.86, P=0.0001 ; \rho(10)=$ $0.22, \quad P=0.53)$ and recovery $(t(7)=10.38, \quad P=0.0001$; $\rho(8)=0.90, P=0.002)$ scans. MRS-derived tissue water T2 also showed group differences across time $(\mathrm{F}(2,32)=3.93$, $P=0.0299)$ : at the binge $(t(19)=5.17, P=0.0001)$ but not baseline or recovery scans, EtOH compared with Dex animals had significantly lower tissue water T2. The EtOH group showed a significant decrease in tissue water T2 between baseline and binge scans $(t(9)=5.32, P=0.0005)$ and a significant increase between binge and recovery scans $(t(7)=5.52, P=0.0009)$.

\section{Binge EtOH Reversibly Modifies Gray Matter Diffusivity in the Thalamus}

Gray matter structures evaluated with DWI metrics included the cortex, dorsal and ventral hippocampi, 

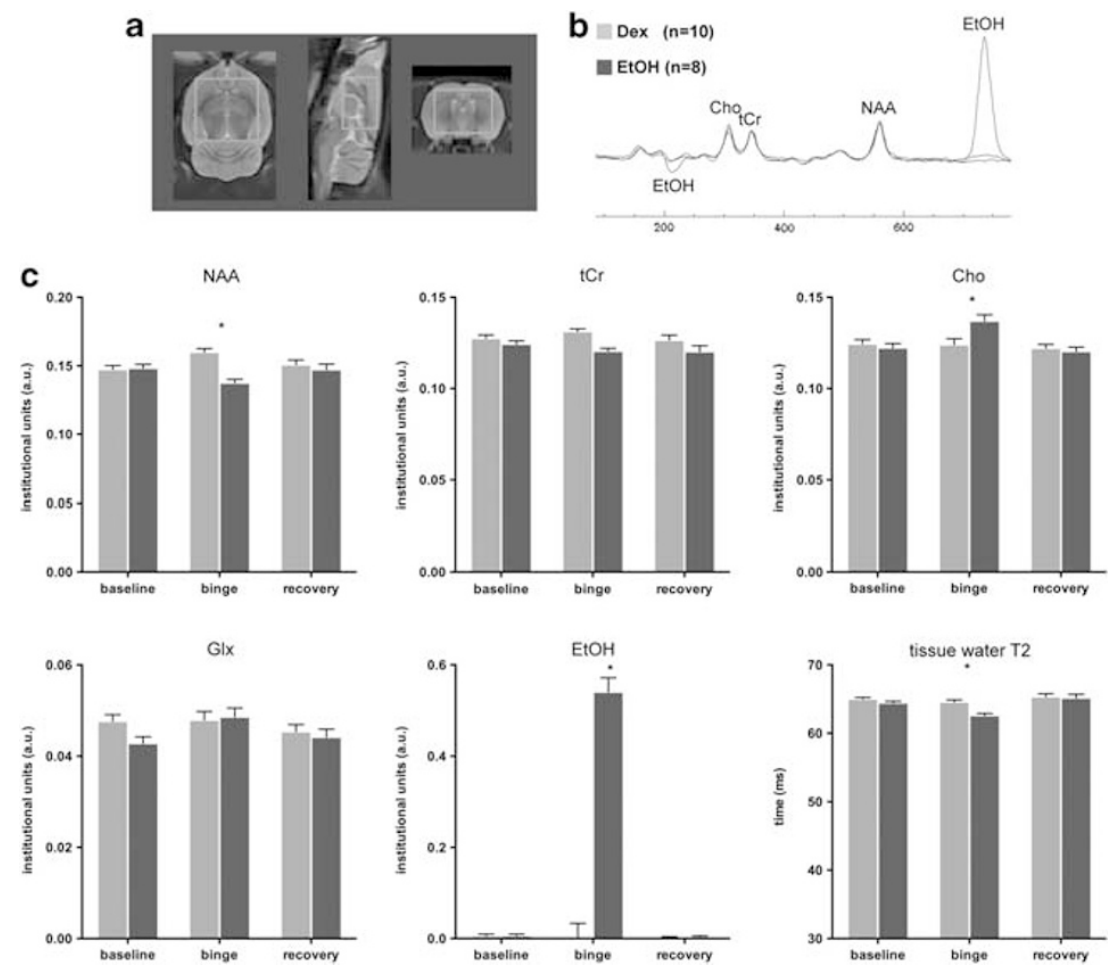

Figure 3 (a) MRS voxel placement in rat axial, sagittal, and coronal planes. (b) Averaged spectra from eight EtOH (red) and ten Dex (gray) animals. (c) Means \pm SEM of the four metabolites, $\mathrm{EtOH}$, and tissue water T2 in EtOH (red) and Dex (gray) animals at baseline, binge, and recovery scans. Cho, choline-containing compounds; Dex, dextrose; EtOH, ethanol; Glx, combined resonances of glutamate and glutamine; MRS, magnetic resonance spectroscopy; NAA, N-acetylasparate; $\mathrm{tCr}$, total Creatine; $* \geqslant 0.05$. The color reproduction of this figure is available on the Neuropsychopharmacology online.

caudate-putamen, and thalamus. For $\mathrm{MD}$, a two-group, repeated-measures ANOVA showed a group-by-time interaction for the thalamus $(\mathrm{F}(2,30)=5.76, \quad P=0.0077$, $\mathrm{GG}=0.0131$ ) (Figure 4 ). In the EtOH group at the binge scan, MD in the thalamus decreased compared with the baseline scan $(t(9)=9.9, P=0.0001)$ and was lower than in the Dex group $(t(18)=5.87, P=0.0001)$. MD levels in the thalamus at the recovery scan, however, had increased to baseline levels in the EtOH group $(t(7)=3.7, P=0.0077)$ and were no longer different from the Dex group $(t(18)=0.036$, $P=0.9714)$. By contrast, ANOVAs for $\mathrm{MD}$ did not reveal group-by-time interactions for cortex $(\mathrm{F}(2,30)=0.0217, P=$ $0.9785)$, dorsal hippocampus $(\mathrm{F}(2,30)=1.8408, P=0.1762)$, ventral hippocampus $(\mathrm{F}(2,30)=1.9950, P=0.1537)$, or caudate-putamen $(\mathrm{F}(2,30)=0.3891, P=0.6810)$.

\section{Relations Between MR Imaging Variables During Binge EtOH Exposure}

To test our hypothesis that ventricular enlargement in response to binge EtOH exposure is related to movement of water out of tissue, we evaluated relations between the MR modality variables and included all 20 animals scanned at the binge time point. Correlations between ventricular volume and the volumes of the dorsal $(r=0.25, P=0.30)$ and ventral hippocampi ( $r=0.20, P=0.41)$, caudate-putamen $(r=0.16, P=0.49)$, and thalamus $(r=-0.14, P=0.56)$ were not forthcoming. There was, however, a negative correlation between MRS-derived tissue water T2 and ventricular volume $(r=-0.6799, P=0.0010$, Figure 5a) and a similar negative correlation between thalamic diffusivity and ventricular volume $(r=-0.5406, P=0.0139$, Figure $5 b)$, suggesting that as tissue water decreases, ventricular volume expands. These relations were not evident at the baseline scan (ventricular volume with tissue water T2: $r=0.5140$, $P=0.0243$, in the unexpected direction; with thalamus MD: $r=0.2466, P=0.3089$ ) nor at the recovery scan (ventricular volume with tissue water T2: $r=-0.0544, P=0.8304$; with thalamus MD: $r=0.1212, P=0.6318$ ).

At the binge scan, lower NAA correlated with lower tissue water T2 $(r=0.7391, P=0.0001$, Figure 5c) and lower thalamic diffusivity $(r=0.6436, P=0.0022$, Figure $5 d)$. By contrast, lower NAA was associated with larger ventricular volume $(r=-0.5507, P=0.0119$, Figure 5e). At the binge scan, Cho levels also correlated with tissue water T2 $(r=-0.5236, P=0.0149$, Figure 5f) and diffusivity in the thalamus ( $r=-0.4697, P=0.0367$, Figure $5 g$ ), but these were negative correlations, such that higher Cho was associated with lower tissue water T2 and lower thalamic diffusivity with a trend for higher Cho correlating with larger ventricular volume $(r=0.4086, \quad P=0.0736$, Figure $5 \mathrm{~h})$. None of the correlations based on NAA or Cho were forthcoming at the baseline or recovery scans.

\section{DISCUSSION}

This longitudinal experiment provides in vivo, multimodal MR evidence for a mechanism of reversible ventricular enlargement. Using our established binge EtOH model to produce ventricular expansion, MRI, at this resolution, found no detectable volume changes in dorsal and ventral 

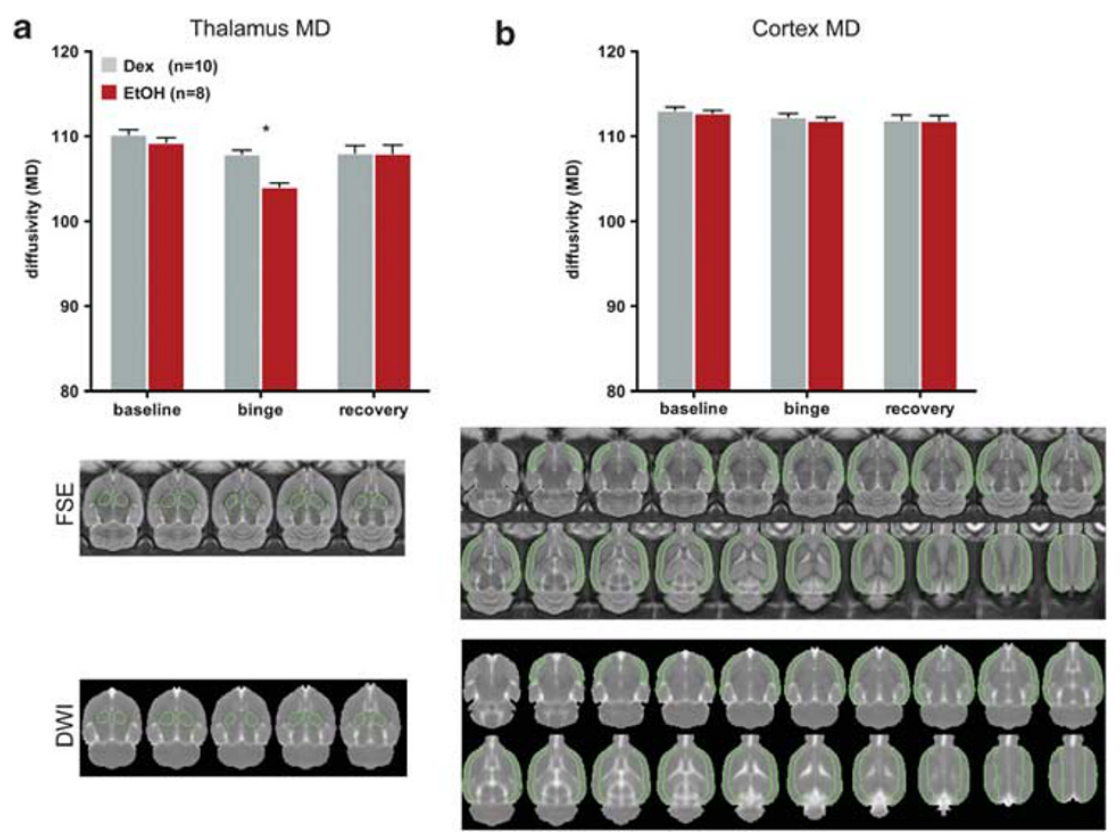

Figure 4 Quantification of MD in (a) thalamus and (b) cortex of EtOH (red) and Dex (gray) animals; figure includes outlines of regions of interest used for quantification of MD on both FSE and DWI images. Dex, dextrose; DWI, diffusion weighted images; EtOH, ethanol; FSE, fast spin-echo images; MD, mean diffusivity. $* P \leqslant 0.05$.

hippocampi, caudate-putamen, or thalamus. MRS of brain tissue revealed increases in Cho and decreases in NAA (Zahr et al, 2010) and tissue water T2. At the same time, DWI in the presence of high BALs showed decreased diffusivity (ie, MD) selective to the thalamus, as recently reported in healthy human volunteers acutely challenged with EtOH (Kong et al, 2012). The levels of all MR parameters returned to baseline with 7 days of recovery. Together, these longitudinal data support a model of fluid movement during acute binge EtOH intoxication and recovery. Shifts of fluid between various brain compartments might explain reversibility of ventricular enlargement observed in humans following recovery from alcohol abuse (Zipursky et al, 1989), anorexia nervosa (Enzmann et al, 1977), and prolonged steroid use (Bentson et al, 1978).

Binge EtOH treatment resulted in average BALs of $292 \mathrm{mg} /$ $\mathrm{dl}$ and ventricular expansion to $122 \%$ of baseline. This pattern of changes is in close agreement with our previous results in which average BALs of $258 \mathrm{mg} / \mathrm{dl}$ were associated with ventricular volume increases to $115 \%$ of baseline (Zahr et al, 2010). In both studies, ventricular size returned to baseline with 7 days of recovery. Enlargement of the lateral ventricles, observed in normal aging (Pfefferbaum et al, 1994; Walhovd et al, 2011), and many pathologies including traumatic brain injury (Bigler and Maxwell, 2011), Alzheimer's disease (Fox and Schott, 2004), and schizophrenia (Sayo et al, 2012), is often interpreted as a marker of atrophy (ie, cell loss) of the surrounding brain regions. In the current study, however, measurement of selective brain regions (ie, dorsal and ventral hippocampi, caudate-putamen, thalamus) did not detect volume changes that could explain ventricular expansion. Furthermore, ventriculomegaly due to atrophy would be difficult to reconcile with rapid recovery of ventricular volume within 1 week.
Mechanisms of ventricular contraction and expansion are poorly understood but are influenced by cerebrospinal fluid (CSF), vascular, and brain tissue properties (Johanson et al, 2008). There is evidence for EtOH effects on CSF (Nixon, 2008) and vasculature (Altura and Altura, 1984), but we propose compensatory (ie, for shifts of fluid between various brain compartments within a closed space) ventriculomegaly is due to changes in brain tissue properties. Specifically, we posit that EtOH causes widespread intracellular water extravasation leading to shrinkage of brain cells and their process. EtOH influences a variety of CNS metabolic processes, but appears to initially affect water movement (Klemm, 1998) by acutely effecting a hyperosmotic environment (Champion et al, 1975). In situations characterized by hyperosmolarity, responses include loss of intracellular water (Pollock and Arieff, 1980) and brain cell shrinkage (Cserr et al, 1987). Excess extracellular fluid is presumed to be quickly drained into the ventricular system to be absorbed by venous sinuses, but excretion of fluid into vasculature of the brain parenchyma has also been proposed (Bloch et al, 2006). Shrinkage of brain cells and their processes (Obernier et al, 2002) might occur in the absence of gross tissue volume loss (Miguel-Hidalgo et al, 2002). Alternatively, tissue volume loss in this study sample may have been too small or too widely distributed to detect in any single region. Thus, another interpretation for the current findings is that regional volume does not change because as neurons lose water and shrink, astrocytes swell (due to the abundant presence on astrocytes of aquaporins involved in cell volume regulation (Yool, 2007)).

Both tissue water T2 and diffusivity are influenced by water mobility (Lei et al, 2003). Tissue water T2 reflects changes in the ratio of bound to unbound water and altered tissue water content (Del Bigio et al, 2011). MD, a measure 

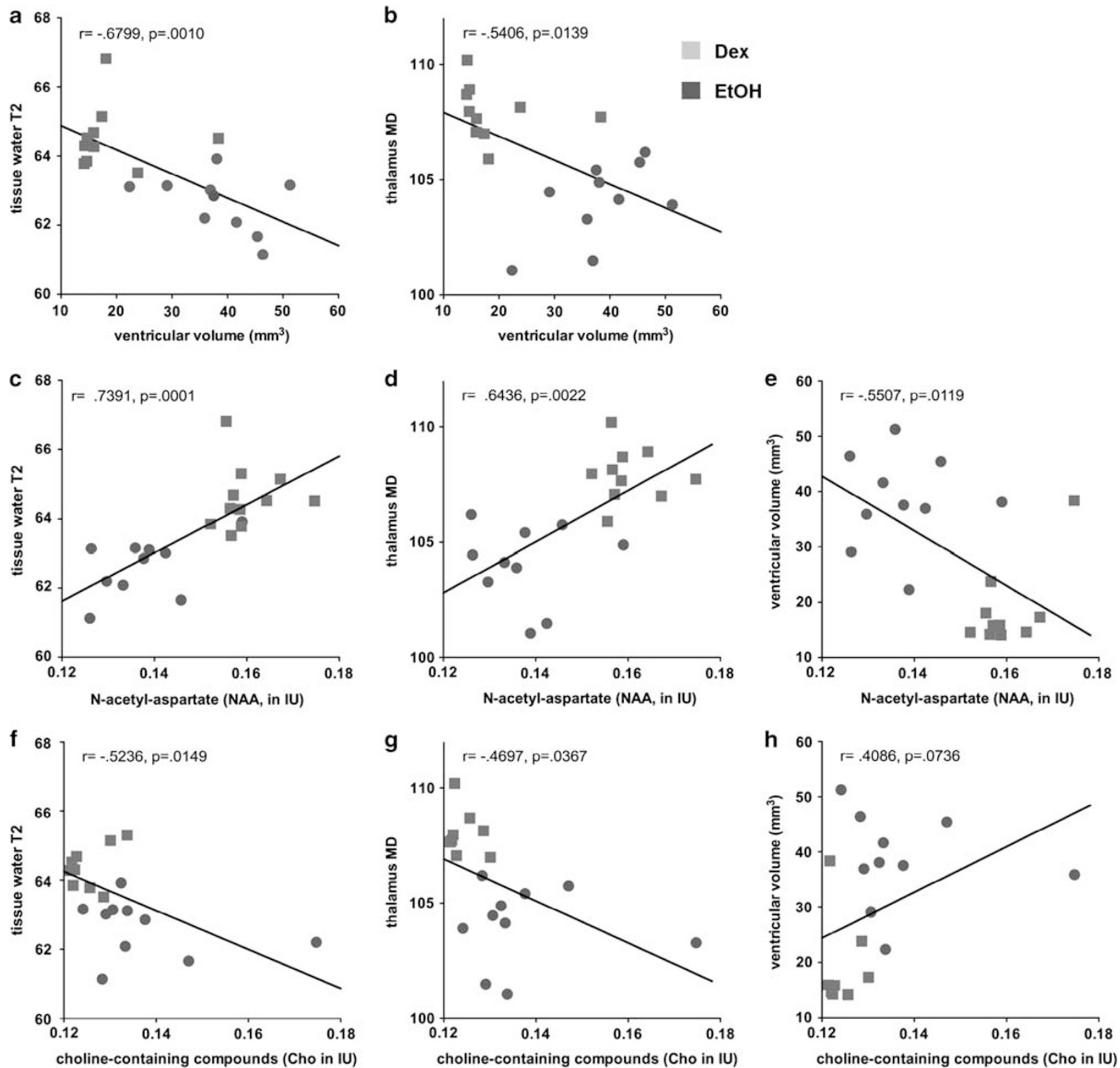

Figure 5 Graphs presenting correlations between various MR variables at the binge time point. Cho, choline-containing compounds; MD, mean diffusivity; MR, magnetic resonance; NAA, N-acetylasparate. The color reproduction of this figure is available on the Neuropsychopharmacology online.

of tissue water diffusivity, is influenced by interactions between water molecules and their surrounding chemical and structural environment (Le Bihan et al, 1986). Together, the correlations between decreased tissue water T2 (ie, Figure 5a) and decreased diffusivity in the thalamus (ie, Figure 5b) with increased ventricular size support the hypothesis that the ventricles enlarge to compensate for water movement out of tissue in the presence of high BALs. Diffusivity changes unique to thalamus might be explained by the selective vulnerability of the thalamus to nutritional effects of EtOH as observed in Wernicke-Korsakoff syndrome (Zahr et al, 2011).

As previously observed (Zahr et al, 2010), binge $\mathrm{EtOH}$ treatment results in transient but significant NAA decreases and Cho increases. The functions of both NAA and Cho remain largely speculative, but our earlier work (ie,
Zahr et al, 2010) highlighted a number of potential interpretations for changes in the levels of these two MRS-detectable metabolites in the context of ventricular enlargement. For example, binge EtOH intoxication could compromise the brain's normal energy utilization. A direct link between energy metabolism and NAA synthesis in mitochondria (Bates et al, 1996) suggests that NAA reductions may be due to energy depletion-induced impairments in mitochondrial NAA synthesis (Moffett et $\mathrm{al}$, 2007). A role for increased Cho as a marker of depressed brain energy utilization is also plausible (Katsura et al, 1993; Trovarelli et al, 1982).

Here, given our additional findings, we propose that both NAA and Cho have a role in osmoregulation. Like its effects on the well-established osmolyte taurine, EtOH increases extracellular NAA levels (Baslow et al, 2000), where NAA 
is rapidly hydrolyzed (Baslow et al, 1999). The positive correlation between NAA and both tissue water T2 (Figure $5 \mathrm{c}$ ), and thalamic diffusivity (Figure $5 \mathrm{~d}$ ) suggests corresponding movement of water and NAA. In compensation for the movement of water and NAA out of neurons, ventricular volume increases (supported by the negative relationship between NAA and ventricular volume, Figure 5e).

The Cho resonance contains contributions from phosphocholine and glycerophosphorylcholine; available evidence suggests that glycerophosphorylcholine, which comprises at least half of the MR-visible peak (Miller et al, 1996), is a cerebral osmolyte (Lien et al, 1990; Videen et al, 1995). Chronic renal failure, for example, associated with a prolonged hyerposmotic state, demonstrates a positive correlation between MRS-derived Cho and serum osmotic pressure (Sasaki et al, 2006). The relations we observed between decreased tissue water T2 (Figure 5f) and thalamic diffusivity (Figure 5g), and increased Cho comport with a role for Cho in compensating for changes in water distribution.

In conclusion, the current study used multiple MR modalities to identify a mechanism of ventricular enlargement. All MR data presented, including decreased tissue water T2, thalamic diffusivity, NAA, and increased Cho, along with relations among these variables and their reversibility with recovery, can be reconciled with a process in which transient, high BALs cause redistribution of water in the brain and compensatory ventriculomegaly. We acknowledge that the average BALs of $\sim 292 \mathrm{mg} / \mathrm{dl}$ achieved in this animal model are more common among experienced than inexperienced users of alcohol (Lahti and Vuori, 2002). We nevertheless believe it is important to understand what might explain the profound and reversible ventricular enlargement we observed following a single cycle of binge $\mathrm{EtOH}$ treatment and recovery before studying a more complex model including repeated binge $\mathrm{EtOH}$ exposure cycles. Over time, repeated EtOH loading may prevent physiological compensations and recovery leading to ventriculomegaly that does reflect lasting pathology. Indeed, repeated changes in water balance may contribute to the demylination (Moffett et al, 2007) observed in chronic alcoholism (Alling and Bostrom, 1980). Such hypotheses can be tested using diffusion tensor imaging to determine the effects of repeated binge EtOH treatment on white matter. We would like to emphasize that this mechanism is in response to acute binge $\mathrm{EtOH}$ intoxication and not meant to explain ventricular enlargement in other conditions in which it is observed. The presentation herein of an alternative mechanism, however, cautions against inferring atrophic processes in ventricular expansion, especially in the absence of concurrent measurement of regional tissue volumes.

\section{ACKNOWLEDGEMENTS}

This study was supported by Grant numbers AA013521INIA, AA005965, AA017168.

\section{DISCLOSURE}

The authors declare no conflict of interest.

\section{REFERENCES}

Adalsteinsson E, Hurd RE, Mayer D, Sailasuta N, Sullivan EV, Pfefferbaum A (2004). In vivo 2D J-resolved magnetic resonance spectroscopy of rat brain with a $3-\mathrm{T}$ clinical human scanner. NeuroImage 22: 381-386.

Alling C, Bostrom K (1980). Demyelination of the mamillary bodies in alcoholism. A combined morphological and biochemical study. Acta Neuropathologica (Berl) 50: 77-80.

Altura BM, Altura BT (1984). Alcohol, the cerebral circulation and strokes. Alcohol (Fayetteville, NY) 1: 325-331.

Baslow MH, Suckow RF, Hungund BL (2000). Effects of ethanol and of alcohol dehydrogenase inhibitors on the reduction of $\mathrm{N}$-acetylaspartate levels of brain in mice in vivo: a search for substances that may have therapeutic value in the treatment of Canavan disease. J Inherit Metab Dis 23: 684-692.

Baslow MH, Suckow RF, Sapirstein V, Hungund BL (1999). Expression of aspartoacylase activity in cultured rat macroglial cells is limited to oligodendrocytes. J Mol Neurosci 13: 47-53.

Bates TE, Strangward M, Keelan J, Davey GP, Munro PMG, Clark JB (1996). Inhibition of $\mathrm{N}$-acetylaspartate production: implications for H-1 MRS studies in vivo. Neuroreport 7: 1397-1400.

Bendszus M, Weijers HG, Wiesbeck G, Warmuth-Metz M, Bartsch AJ, Engels $S$ et al (2001). Sequential MR imaging and proton MR spectroscopy in patients who underwent recent detoxification for chronic alcoholism: correlation with clinical and neuropsychological data. Amer J Neuroradiol 22: 1926-1932.

Bengzon J, Mohapel P, Ekdahl CT, Lindvall O (2002). Neuronal apoptosis after brief and prolonged seizures. Prog Brain Res 135: 111-119.

Bentson J, Reza M, Winter J, Wilson G (1978). Steroids and apparent cerebral atrophy on computed tomography scans. J Comput Assist Tomogr 2: 16-23.

Bigler ED, Maxwell WL (2011). Neuroimaging and neuropathology of TBI. NeuroRehabilitation 28: 63-74.

Bloch O, Auguste KI, Manley GT, Verkman AS (2006). Accelerated progression of kaolin-induced hydrocephalus in aquaporin-4deficient mice. J Cereb Blood Flow Metab 26: 1527-1537.

Bottomley PA (1984). Selective volume method for performing localized NMR spectroscopy and NMR chemical shift imaging. US Patent 4,480,228; 30 October 1984.

Butzkueven H, Kolbe SC, Jolley DJ, Brown JY, Cook MJ, van der Mei IA et al (2008). Validation of linear cerebral atrophy markers in multiple sclerosis. J Clin Neurosci 15: 130-137.

Champion HR, Baker SP, Benner C, Fisher R, Caplan YH, Long WB et al (1975). Alcohol intoxication and serum osmolality. Lancet 1: 1402-1404.

Chronik B, Alejski A, Rutt BK (2000). Design and fabrication of a three-axis multilayer gradient coil for magnetic resonance microscopy of mice. MAGMA 10: 131-146.

Cook PA, Bai Y, Nedjati-Gilani S, Seunarine KK, Hall MG, Parker GJ et al (eds) (2006). Camino: Open-Source Diffusion-MRI Reconstruction and Processing. International Society for Magnetic Resonance in Medicine: Seattle, WA.

Cserr HF, DePasquale M, Patlak CS (1987). Regulation of brain water and electrolytes during acute hyperosmolality in rats. Am J Physiol 253(3 Pt 2): F522-F529.

De Stefano N, Bartolozzi ML, Guidi L, Stromillo ML, Federico A (2005). Magnetic resonance spectroscopy as a measure of brain damage in multiple sclerosis. J Neurol Sci 233: 203-208.

Del Bigio MR, Slobodian I, Schellenberg AE, Buist RJ, Kemp-Buors TL (2011). Magnetic resonance imaging indicators of bloodbrain barrier and brain water changes in young rats with kaolininduced hydrocephalus. Fluids Barriers CNS 8: 22.

Enzmann DR, Lane B (1977). Cranial computed tomography findings in anorexia nervosa. J Comput Assist Tomogr 1: 410-414.

Fox NC, Schott JM (2004). Imaging cerebral atrophy: normal ageing to Alzheimer's disease. Lancet 363: 392-394. 
Jackson DC, Irwin W, Dabbs K, Lin JJ, Jones JE, Hsu DA et al (2011). Ventricular enlargement in new-onset pediatric epilepsies. Epilepsia 52: 2225-2232.

Johanson CE, Duncan JA 3rd, Klinge PM, Brinker T, Stopa EG, Silverberg GD (2008). Multiplicity of cerebrospinal fluid functions: New challenges in health and disease. Cerebrospinal Fluid Res 5: 10.

Katsura K, Rodriguez de Turco EB, Folbergrova J, Bazan NG, Siesjo BK (1993). Coupling among energy failure, loss of ion homeostasis, and phospholipase $\mathrm{A} 2$ and $\mathrm{C}$ activation during ischemia. J Neurochem 61: 1677-1684.

Klemm WR (1998). Biological water and its role in the effects of alcohol. Alcohol (Fayetteville, NY) 15: 249-267.

Kong LM, Zheng WB, Lian GP, Zhang HD (2012). Acute effects of alcohol on the human brain: diffusion tensor imaging study. AJNR 33: 928-934.

Lahti RA, Vuori E (2002). Fatal alcohol poisoning: medico-legal practices and mortality statistics. Forensic Sci Int 126: 203-209.

Le Bihan D, Breton E, Lallemand D, Grenier P, Cabanis E, LavalJeantet M (1986). MR imaging of intravoxel incoherent motions: application to diffusion and perfusion in neurologic disorders. Radiology 161: 401-407.

Lei H, Zhang Y, Zhu XH, Chen W (2003). Changes in the proton T2 relaxation times of cerebral water and metabolites during forebrain ischemia in rat at 9.4T. Magn Reson Med 49: 979-984.

Lien YH, Shapiro JI, Chan L (1990). Effects of hypernatremia on organic brain osmoles. J Clin Invest 85: 1427-1435.

Mader I, Rauer S, Gall P, Klose U (2008). (1)H MR spectroscopy of inflammation, infection and ischemia of the brain. Eur J Radiol 67: 250-257.

Majchrowicz E (1975). Induction of physical dependence upon ethanol and the associated behavioral changes in rats. Psychopharmacologia 43: 245-254.

Miguel-Hidalgo JJ, Wei J, Andrew M, Overholser JC, Jurjus G, Stockmeier CA et al (2002). Glia pathology in the prefrontal cortex in alcohol dependence with and without depressive symptoms. Biol Psychiatry 52: 1121-1133.

Miller BL, Chang L, Booth R, Ernst T, Cornford M, Nikas D et al (1996). In vivo $1 \mathrm{H}$ MRS choline: correlation with in vitro chemistry/histology. Life Sci 58: 1929-1935.

Moffett JR, Ross B, Arun P, Madhavarao CN, Namboodiri AM (2007). N-Acetylaspartate in the CNS: from neurodiagnostics to neurobiology. Prog Neurobiol 81: 89-131.

Neeman M, Freyer JP, Sillerud LO (1991). A simple method for obtaining cross-term-free images for diffusion anisotropy studies in NMR microimaging. Magn Reson Med 21: 138-143.

Nixon PF (2008). Glutamate export at the choroid plexus in health, thiamin deficiency, and ethanol intoxication: review and hypothesis. Alcohol Clin Exp Res 32: 1339-1349.

Obernier JA, Bouldin TW, Crews FT (2002). Binge ethanol exposure in adult rats causes necrotic cell death. Alcohol Clin Exp Res 26: 547-557.
Pfefferbaum A, Adalsteinsson E, Sullivan EV (2004). In vivo structural imaging of the rat brain with a $3-\mathrm{T}$ clinical human scanner. J Magn Reson Imaging 20: 779-785.

Pfefferbaum A, Mathalon DH, Sullivan EV, Rawles JM, Zipursky RB, Lim KO (1994). A quantitative magnetic resonance imaging study of changes in brain morphology from infancy to late adulthood. Arch Neurol 51: 874-887.

Pfefferbaum A, Rosenbloom MJ, Rohlfing T, Adalsteinsson E, Kemper CA, Deresinski S et al (2006). Contribution of alcoholism to brain dysmorphology in HIV infection: effects on the ventricles and corpus callosum. Neuroimage 33: 239-251.

Pollock AS, Arieff AI (1980). Abnormalities of cell volume regulation and their functional consequences. Am J Physiol 239: F195-F205.

Sasaki O, Hattori N, Nakahama H, Inoue N, Nakamura S, Inenaga $\mathrm{T}$ et al (2006). Positive correlations between cerebral choline and renal dysfunction in chronic renal failure. Neuroradiology 48: 300-306.

Sayo A, Jennings RG, Van Horn JD (2012). Study factors influencing ventricular enlargement in schizophrenia: a 20 year follow-up meta-analysis. NeuroImage 59: 154-167.

Symonds LL, Archibald SL, Grant I, Zisook S, Jernigan TL (1999). Does an increase in sulcal or ventricular fluid predict where brain tissue is lost? J Neuroimaging 9: 201-209.

Trovarelli G, De Medio GE, Montanini I (1982). The influence of CDP-choline on brain lipid metabolism during ischemia. Farmaco [Sci] 37: 663-668.

Videen JS, Michaelis T, Pinto P, Ross BD (1995). Human cerebral osmolytes during chronic hyponatremia. A proton magnetic resonance spectroscopy study. J Clin Invest 95: 788-793.

Walhovd KB, Westlye LT, Amlien I, Espeseth T, Reinvang I, $\mathrm{Raz} \mathrm{N}$ et al (2011). Consistent neuroanatomical age-related volume differences across multiple samples. Neurobiol Aging 32: 916-932.

Yool AJ (2007). Aquaporins: multiple roles in the central nervous system. Neuroscientist 13: 470-485.

Zahr NM, Kaufman KL, Harper CG (2011). Clinical and pathological features of alcohol-related brain damage. Nat Rev Neurol 7: 284-294.

Zahr NM, Mayer D, Rohlfing T, Hasak M, Hsu O, Vinco S et al (2010). Brain Injury and recovery following binge ethanol: evidence from in vivo magnetic resonance spectroscopy. Biol Psychiatry 67: 846-854.

Zahr NM, Mayer D, Vinco S, Orduna J, Luong R, Sullivan EV et al (2009). In vivo evidence for alcohol-induced neurochemical changes in rat brain without protracted withdrawal, pronounced thiamine deficiency, or severe liver damage. Neuropsychopharmacology 34: 1427-1442.

Zipursky RB, Lim KO, Pfefferbaum A (1989). MRI study of brain changes with short term abstinence from alcohol. Alcoholism Clin Exp Res 13: 664-666.

Supplementary Information accompanies the paper on the Neuropsychopharmacology website (http://www.nature.com/npp) 